\title{
Terminal Olefins from Aldehydes Through Enol Triflate Reduction
}

Sushil K. Pandey, Andrew E. Greene, and Jean-François Poisson*

Département de Chimie Moléculaire (SERCO) CNRS, UMR-5250, ICMG FR-2607, Université Joseph Fourier

Jean-francois.poisson@ujf-grenoble.fr

\section{SUPPORTING INFORMATION}

General Information $\quad$ S1

$\begin{array}{ll}\text { Method A } & \text { S2 }\end{array}$

Method B $\quad$ S2

3-(Benzo[d][1,3]dioxol-5-yl)-2-methylprop-1-enyl Trifluoromethanesulfonate (3a') $\quad$ S2

5-(2-Methylallyl)benzo[d][1,3]dioxole (4a) $\quad$ S3

2-Methylundec-1-ene (4b) $\quad$ S3

1,3,3-Trimethyl-2-(3-methylbut-3-enyl)cyclohex-1-ene (4c) $\quad$ S4

$\begin{array}{ll}\text { Prop-1-en-2-ylbenzene (4d) } & \text { S4 }\end{array}$

$\begin{array}{ll}\text { Dodec-1-ene (4e) } & \text { S4 }\end{array}$

$\begin{array}{ll}\text { Allylbenzene (4f) } & \text { S5 }\end{array}$

${ }^{1} \mathbf{H}$ NMR of $3 \mathbf{a}^{\prime} \quad$ S6

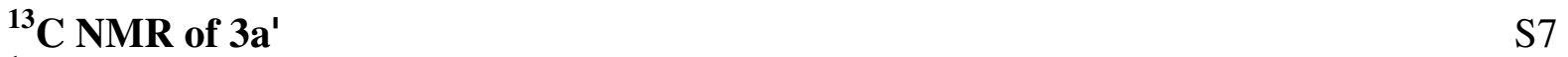

${ }^{1} \mathbf{H}$ NMR of $4 \mathbf{a} \quad$ S8

${ }^{13} \mathrm{C}$ NMR of $4 \mathrm{a} \quad \mathrm{S} 9$

${ }^{1} \mathrm{H}$ NMR of $4 \mathrm{~b} \quad \mathrm{~S} 10$

${ }^{13} \mathrm{C}$ NMR of $4 b \quad$ S11

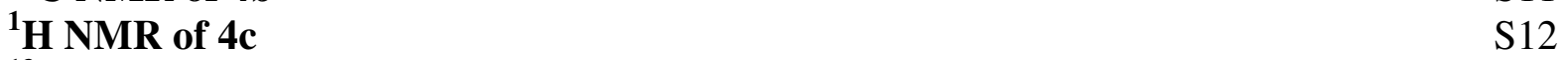

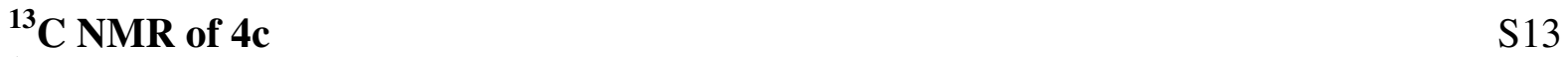

${ }^{1}$ H NMR of 4d $\quad$ S14

${ }^{13}$ C NMR of 4d $\quad$ S15

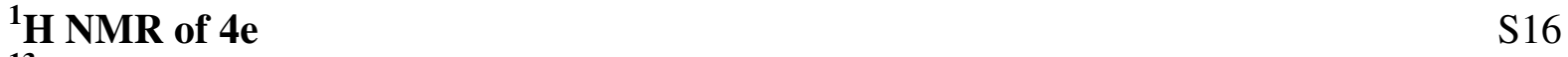

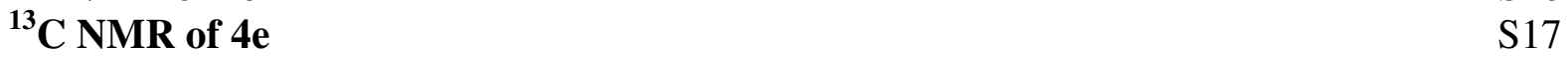

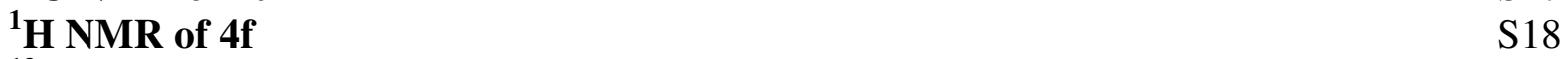

${ }^{13}$ C NMR of $4 \mathbf{f} \quad$ S19

Reactions were generally carried out under argon in oven-dried glassware.

Standard inert atmosphere techniques were used in handling all air and moisture sensitive reagents. Dry THF was obtained by filtration through activated molecular sieves and dry DMF by filtration through activated aluminium oxide. Thin-layer chromatography was performed on $(0.2 \mathrm{~mm})$ silica sheets, which were visualized under ultraviolet light and by 
heating the plate after treatment with phosphomolybdic acid in ethanol, a $p$-anisaldehyde staining solution $(80 \mathrm{~mL}$ of $95 \%$ ethanol, $2.9 \mathrm{~mL}$ of sulfuric acid, $0.86 \mathrm{~mL}$ of acetic acid, $2.1 \mathrm{~mL}$ of $p$-anisaldehyde), ninhydrin in ethanol, ceric ammonium molybdate in ethanol, or basic, aqueous $\mathrm{KMnO}_{4}$. Silica gel (0.040-0.063 mm) was employed for flash column chromatography. A Fourier transform infrared spectrometer was used to record IR spectra. ${ }^{1} \mathrm{H}$ NMR and ${ }^{13} \mathrm{C}$ NMR spectra were recorded on either a 300 or a $500 \mathrm{MHz}$ apparatus. All shifts for ${ }^{1} \mathrm{H}$ spectra were referenced to the residual solvent peak and are reported in ppm. When ambiguous, proton and carbon assignments were established through COSY, HMQC, and/or DEPT experiments. Mass spectra were recorded using either DCI (ammonia/isobutane 63/37), EI, or ESI techniques. HRMS were recorded on an Orbitrap apparatus (ESI).

Method A: To a cold $\left(-15^{\circ} \mathrm{C}\right)$ solution of tert-BuOK $(1.2 \mathrm{mmol})$ in dry THF is added aldehyde 3 (1.0 mmol). After stirring for $5 \mathrm{~min}$, Comins' reagent $(1.3 \mathrm{mmol})$ is added and the reaction mixture is allowed to stir for an additional $40 \mathrm{~min}$. Aqueous workup, followed by flash chromatography on silica gel, affords the corresponding triflate. Reduction of the triflate is realized under the conditions described in method C.

Method B: Same as method C, but the intermediate triflate is purified by flash chromatography on silica gel.

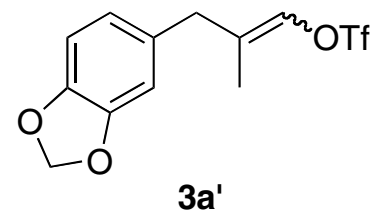

3-(Benzo[d][1,3]dioxol-5-yl)-2-methylprop-1-enyl Trifluoromethanesulfonate (3a'). Obtained by method B. IR (film) 3099, 2896, 2775, 1681, 1610, 1503, 1489, 1418, 1244, $1212,1144,1041,995,867 \mathrm{~cm}^{-1} ;{ }^{1} \mathrm{H}$ NMR $\left(\mathrm{CDCl}_{3}, 300 \mathrm{MHz}, 5: 4\right.$ mixture $) \delta 6.75(\mathrm{~d}, J=$ $8.3 \mathrm{~Hz}, 1 \mathrm{H}), 6.63$ (m, 2H), 6.51 (brs, 1H), 5.94 and 5.93 (s, 2H), 3.41 and 3.21 (s, 2H), 1.69 and $1.60(\mathrm{~d}, J=1.5 \mathrm{~Hz}, 3 \mathrm{H}) ;{ }^{13} \mathrm{C}$ NMR $\left(\mathrm{CDCl}_{3}, 75 \mathrm{MHz}, 5: 4\right.$ mixture) $147.9,146.5$, 146.3, 131.7, 130.7, 130.6, 130.4, 129.6, 128.5, 125.0, 121.7, 121.5, 120.7, 116.5, 112.2, 
108.9, 108.2, 101.0, 100.9, 39.1, 35.0, 16.4, 13.4; EIMS m/z $324\left(\mathrm{M}^{+}\right), 191,174,163$, 133, 105 (100\%), 77. HRMS (LTQ-Orbitrap, ESI) Calcd. for $\mathrm{C}_{12} \mathrm{H}_{11} \mathrm{~F}_{3} \mathrm{NaO}_{5} \mathrm{~S}: 347.0177$. Found $347.0178\left(\mathrm{M}+\mathrm{Na}^{+}\right)$.

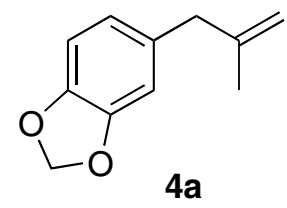

5-(2-Methylallyl)benzo[d][1,3]dioxole (4a). Obtained by method $\mathrm{C}$ starting from aldehyde 3a (100 mg, $0.51 \mathrm{mmol}$ ), affording 4 a (57 $\mathrm{mg}, 63 \%$ over two steps) as a colorless oil: IR (film) 3072, 2969, 2895, 2775, 1651, 1486, 1434, 1240, 1041, 926, $\mathrm{cm}^{-}$ 1; ${ }^{1} \mathrm{H}$ NMR $\left(\mathrm{CDCl}_{3}, 300 \mathrm{MHz}\right)$ 8 6.80-6.64 (m, 3H), 5.93 (s, 2H), 4.82 (s, 1H), 4.76 (s, 1H), 3.14 (s, 2H), 1.69 (s, 3H); $\left.{ }^{13} \mathrm{C} \mathrm{NMR} \mathrm{(} \mathrm{CDCl}_{3}, 75 \mathrm{MHz}\right)$ 147.5, 145.8, 145.1, 133.5, 121.7, 111.7, 109.2, 107.9, 100.7, 44.3, 21.8; EIMS m/z $176\left(\mathrm{M}^{+}\right), 161,146,135,131$, 117, 103, 77. HRMS (LTQ-Orbitrap, ESI) Calcd. for $\mathrm{C}_{11} \mathrm{H}_{13} \mathrm{O}_{2}:$ 177.0915. Found $177.0910\left(\mathrm{M}+\mathrm{H}^{+}\right)$.

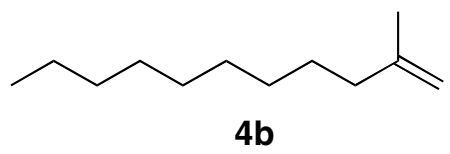

2-Methylundec-1-ene (4b). ${ }^{\mathbf{T}} \quad$ Obtained by method C starting from $\mathbf{3 b}$ (200 mg, 1.49 mmol), affording $\mathbf{4 b}$ (117 mg, 64\% over two steps) as a colorless oil: IR (film) 2958, 2918, 2878, 2849, 1651, 1457, 1240, 1075, 1001, $881 \mathrm{~cm}^{-1}$; ${ }^{1} \mathrm{H} \mathrm{NMR}\left(\mathrm{CDCl}_{3}, 300 \mathrm{MHz}\right)$ $\delta 4.69(\mathrm{~s}, 1 \mathrm{H}), 4.67(\mathrm{~s}, 1 \mathrm{H}), 2.01(\mathrm{t}, J=7.9 \mathrm{~Hz}, 2 \mathrm{H}), 1.72(\mathrm{~s}, 3 \mathrm{H}), 1.52-1.25(\mathrm{~m}, 14 \mathrm{H})$, $0.90(\mathrm{t}, J=6.8 \mathrm{~Hz}, 3 \mathrm{H}) ;{ }^{13} \mathrm{C} \mathrm{NMR}\left(\mathrm{CDCl}_{3}, 75 \mathrm{MHz}\right) 146.1,109.4,37.8,31.9,29.63$, 29.60, 29.3, 28.1, 27.6, 22.6, 22.3, 14.0; MS (DCI, $\mathrm{NH}_{3} /$ isobutane) $\mathrm{m} / 2.186\left(\mathrm{M}+\mathrm{NH}_{4}\right)^{+}$, $169(\mathrm{M}+\mathrm{H})^{+}, 151,109$.

\footnotetext{
${ }^{1}$ Commercially available from Acros.
} 


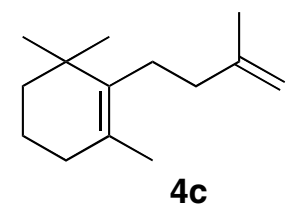

1,3,3-Trimethyl-2-(3-methylbut-3-enyl)cyclohex-1-ene (4c). ${ }^{2}$ Obtained by method A, starting from 3c (100 mg, $0.48 \mathrm{mmol}$ ), affording $4 \mathbf{c}$ (49 $\mathrm{mg}$, 53\% over two steps) as a colorless oil. IR (film) 3072, 2966, 2923, 2867, 1651, 1452, 1445, 1372, 139, 1207 , 1071, $885 \mathrm{~cm}^{-1} ;{ }^{1} \mathrm{H}$ NMR $\left(\mathrm{CDCl}_{3}, 300 \mathrm{MHz}\right) \delta 4.70(\mathrm{~s}, 2 \mathrm{H}), 2.17-2.02(\mathrm{~m}, 4 \mathrm{H}), 1.92(\mathrm{t}, J$ $=6.4 \mathrm{~Hz}, 2 \mathrm{H}), 1.76(\mathrm{~s}, 3 \mathrm{H}), 1.61(\mathrm{~s}, 3 \mathrm{H}), 1.60-1.53(\mathrm{~m}, 2 \mathrm{H}), 1.46-1.40(\mathrm{~m}, 2 \mathrm{H}), 1.00(\mathrm{~s}$, $6 \mathrm{H}) ;{ }^{13} \mathrm{C} \mathrm{NMR}\left(\mathrm{CDCl}_{3}, 75 \mathrm{MHz}\right) 146.8,137.0,127.0,109.0,39.8,38.4,34.9,32.7,28.5$ $\left(2 \mathrm{xCH}_{3}\right), 27.3,22.5,19.7,19.5$.

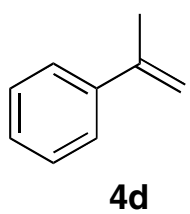

Prop-1-en-2-ylbenzene (4d). ${ }^{3}$ Obtained by procedure C, starting from 3d (200 mg, 1.49 mmol), affording $\mathbf{4 d}$ (115 mg, 65\% over two steps) as a volatile, colorless oil: IR (film) 2952, 2866, 1600, 1457, 1417, 1235, 1075, $1001 \mathrm{~cm}^{-1} ;{ }^{1} \mathrm{H} \mathrm{NMR}\left(\mathrm{CDCl}_{3}, 300 \mathrm{MHz}\right)$ $\delta$ 7.42-7.35 (m, 2H), 7.28-7.12 (m, 3H), $5.28(\mathrm{~s}, 1 \mathrm{H}), 5.03-4.96(\mathrm{~m}, 1 \mathrm{H}), 2.07(\mathrm{~s}, 3 \mathrm{H}) ;{ }^{13} \mathrm{C}$ NMR $\left(\mathrm{CDCl}_{3}, 75 \mathrm{MHz}\right) \delta 143.3,141.3,128.2,127.3,125.5,112.3,21.7$; MS (DCI, $\mathrm{NH}_{3} /$ isobutane) $m / z, 119(\mathrm{M}+\mathrm{H})^{+}(100 \%), 108,91$.

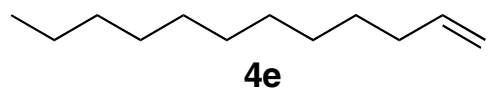

Dodec-1-ene (4e). ${ }^{3}$ Obtained by procedure C, starting from $3 \mathbf{e}(200 \mathrm{mg}, 1.08 \mathrm{mmol})$, affording 4e (132 mg, 72\% over two steps) as a volatile, colorless oil: IR (film) 2957, 2925, 2854, 1642, 1464, 1379, 991, $906 \mathrm{~cm}^{-1} ;{ }^{1} \mathrm{H} \mathrm{NMR}\left(\mathrm{CDCl}_{3}, 300 \mathrm{MHz}\right) \delta 5.88-5.75$

\footnotetext{
${ }^{2}$ Pelz, J.; Bauer, K. Unsaturated Alicyclic Compounds. D.E. Patent 1,235,309, March 2, 1967 [Chem. Abstr. 1967, 67, 22049].

${ }^{3}$ Commercially available from Sigma Aldrich.
} 
(m, 1H), 5.06-4.90 (m, 2H), $2.04(\mathrm{q}, J=7.7 \mathrm{~Hz}, 2 \mathrm{H}), 1.45-1.23(\mathrm{~m}, 16 \mathrm{H}), 0.88(\mathrm{t}, J=6.9$ $\mathrm{Hz}, 3 \mathrm{H}) ;{ }^{13} \mathrm{C} \mathrm{NMR}\left(\mathrm{CDCl}_{3}, 75 \mathrm{MHz}\right) 139.0,114.0,33.8,32.2,31.9$, 29.6, 29.5, 29.4, 29.2, 29.0, 22.7, 14.0; MS (DCI, $\mathrm{NH}_{3}$ /isobutane) $m / z .186\left(\mathrm{M}+\mathrm{NH}_{4}\right)^{+}(100 \%), 123,109$.

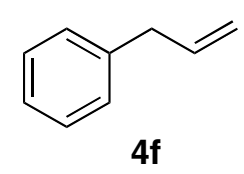

Allylbenzene (4f). ${ }^{4}$ Obtained by procedure C (reduction performed at $35{ }^{\circ} \mathrm{C}$ ), starting from $3 f$ (200 mg, $1.49 \mathrm{mmol})$, affording $\mathbf{4 f}$ (124 mg, 70\% over two steps) as a volatile, colorless oil. IR (film) 3085, 3062, 3026, 2973, 2903, 1641, 1601, 1492, 1455, 1071, 995 $\mathrm{cm}^{-1} ;{ }^{1} \mathrm{H}$ NMR $\left(\mathrm{CDCl}_{3}, 300 \mathrm{MHz}\right) \delta$ 7.27-7.08 (m, 5H), 5.98-5.83 (m, 1H), 5.06-4.96 (m, $2 \mathrm{H}), 3.33(\mathrm{~d}, J=6.6 \mathrm{~Hz}, 2 \mathrm{H}) ;{ }^{13} \mathrm{C} \mathrm{NMR}\left(\mathrm{CDCl}_{3}, 75 \mathrm{MHz}\right) \delta 139.9,137.3,128.5,128.3$, 126.0, 115.6, 40.2; MS (DCI, $\mathrm{NH}_{3} /$ isobutane) $m / z$ 118, 91 (100\%).

${ }^{4}$ Zhang, Q.; Lu, X.; Han, X. J. Org. Chem. 2001, 66, 7676-7684. 


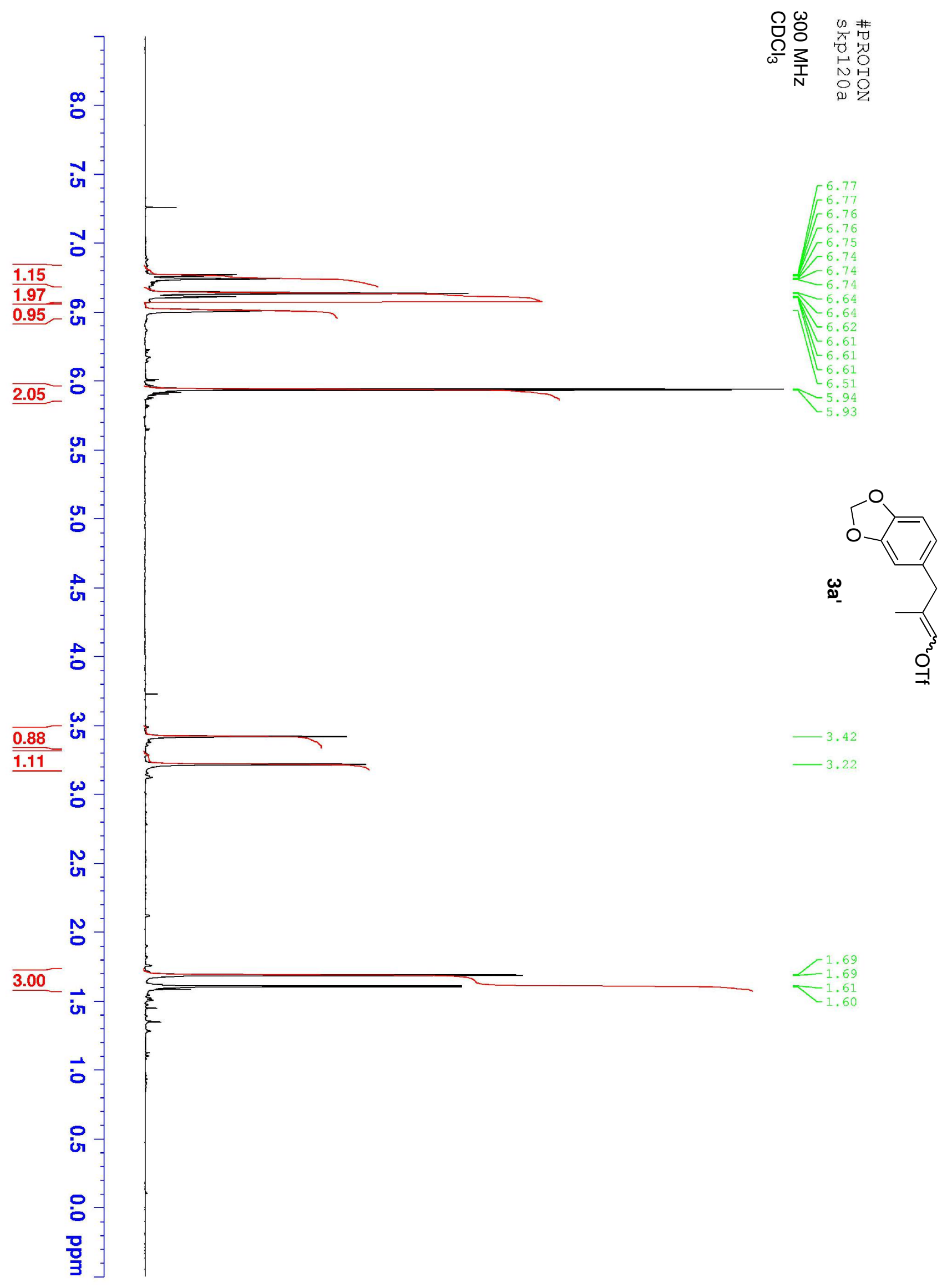




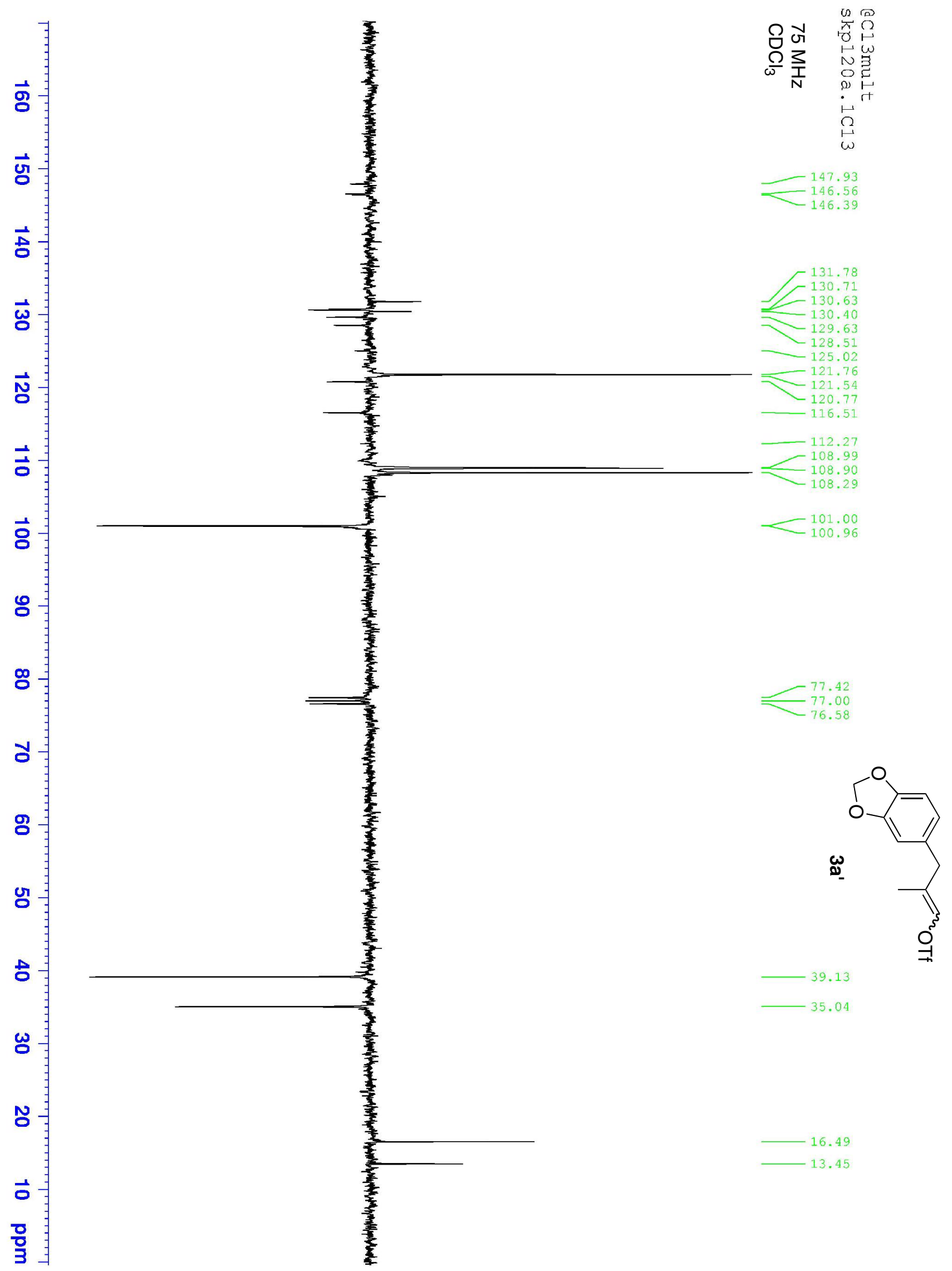




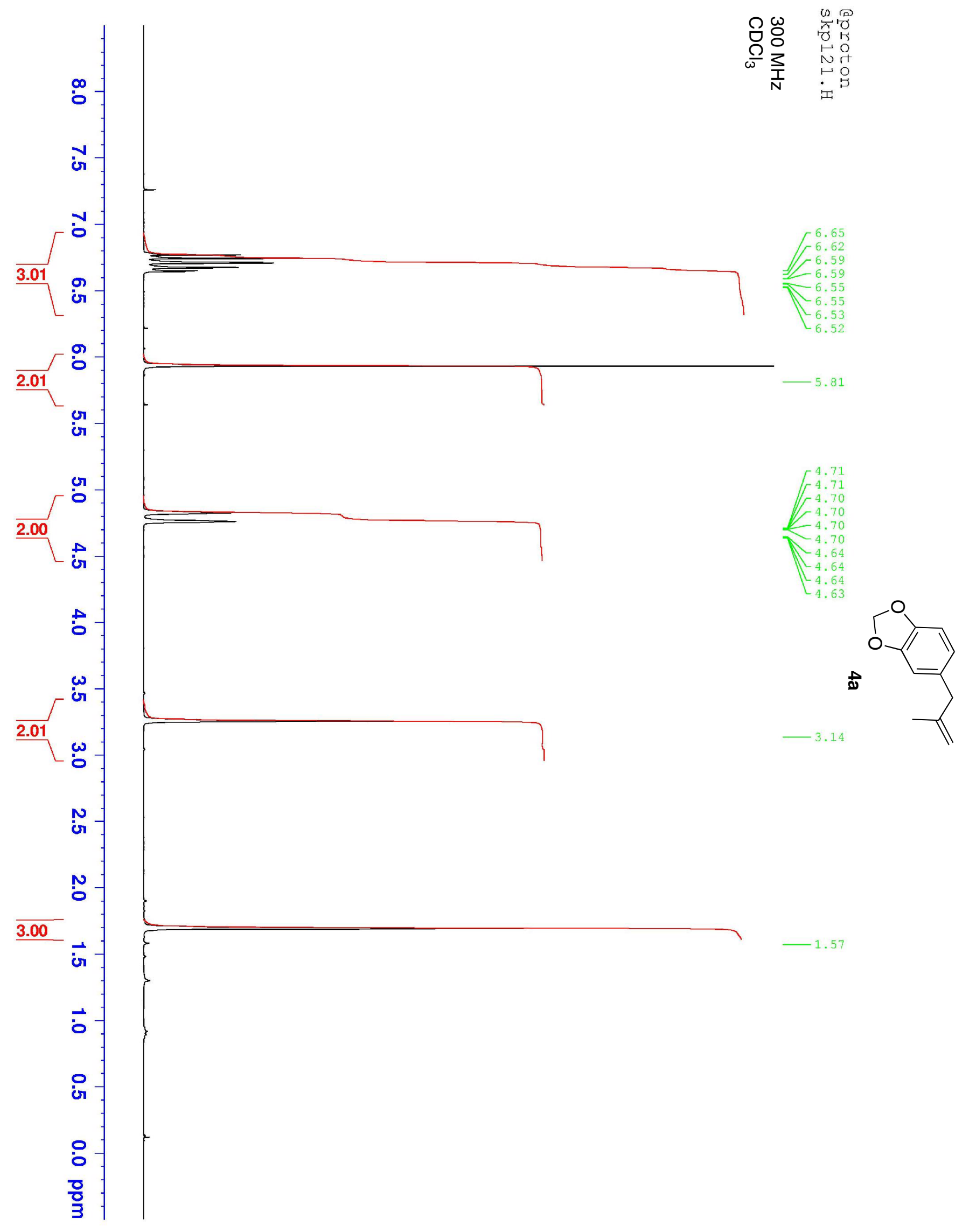




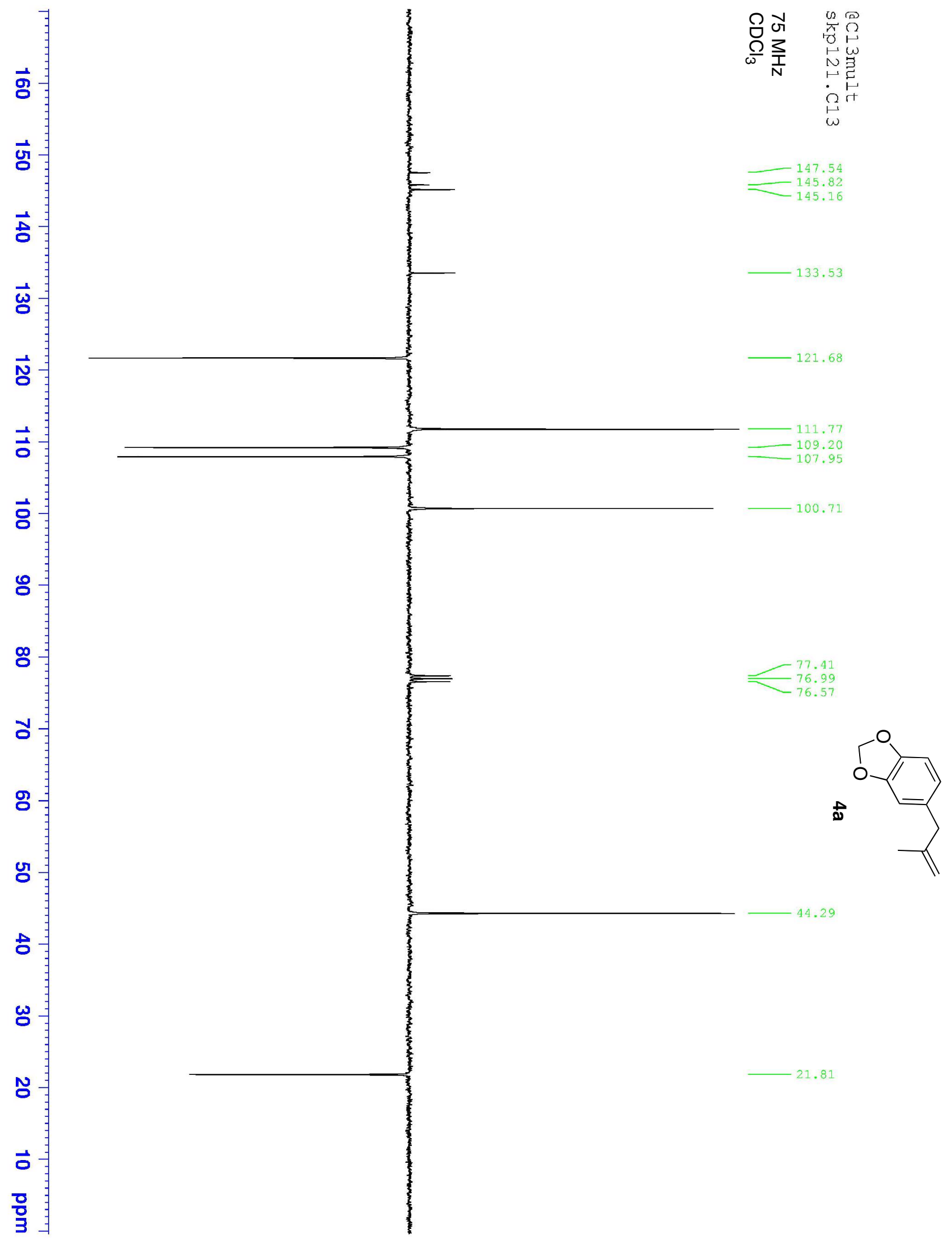




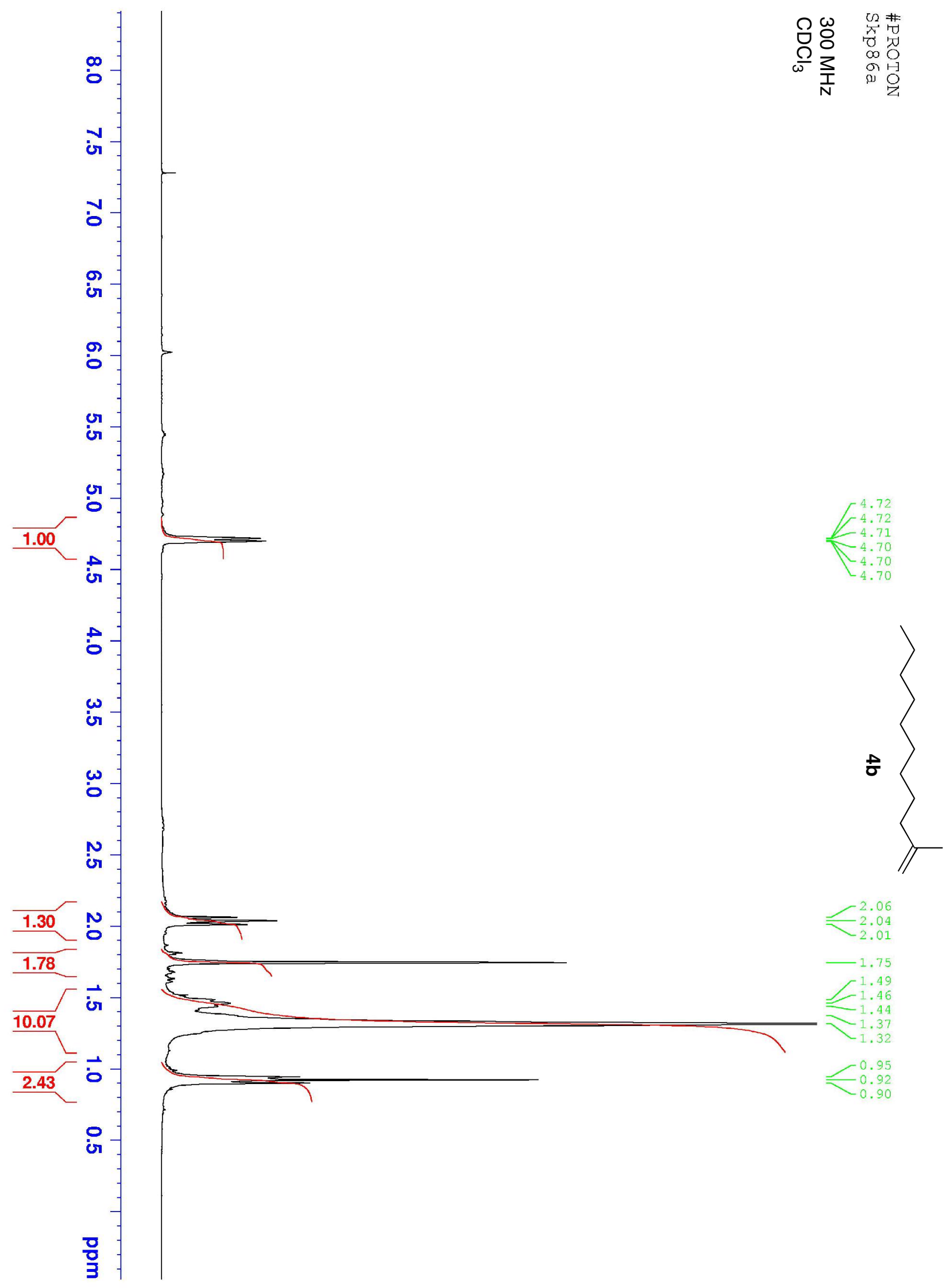




$$
\|
$$




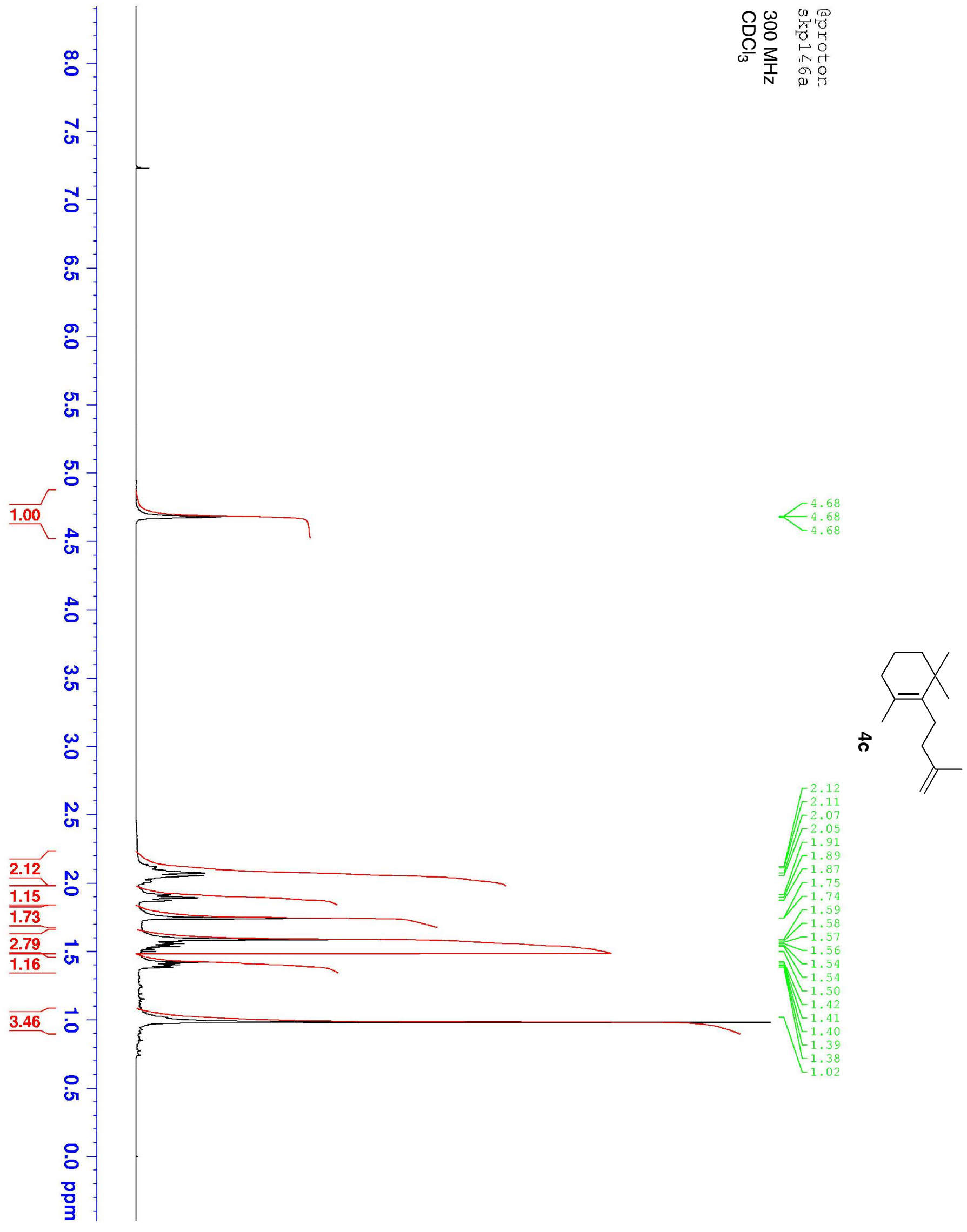




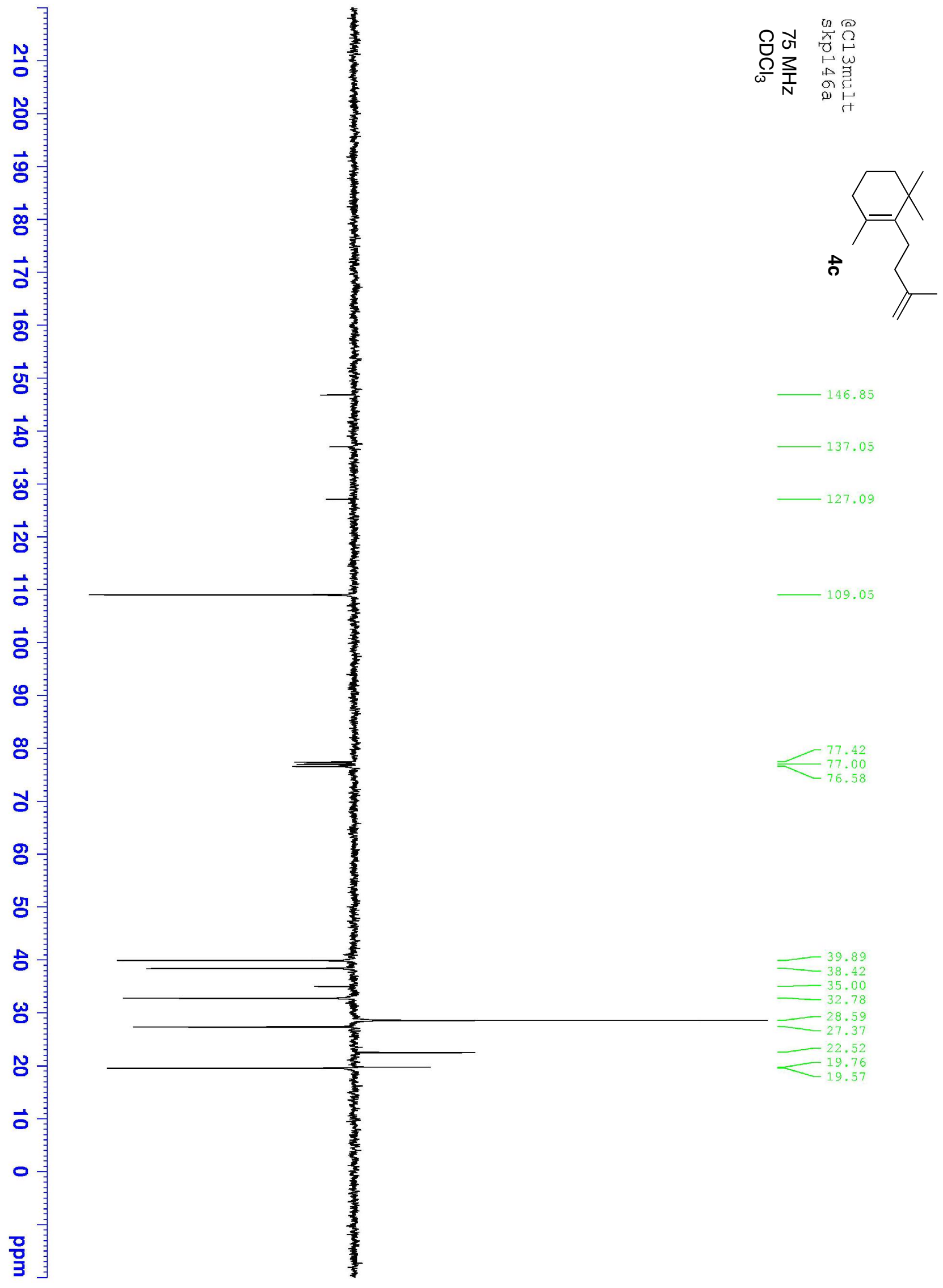




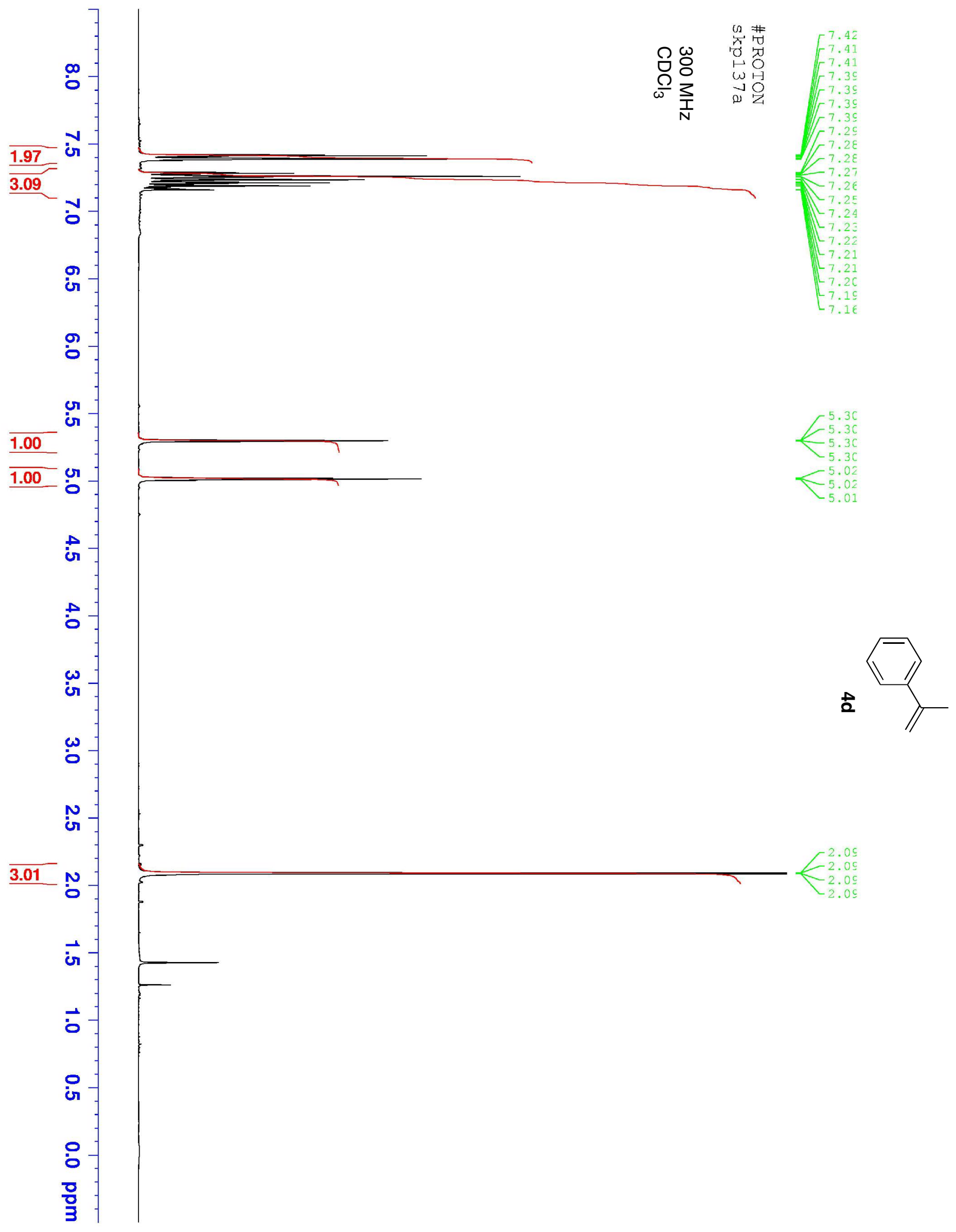




$$
H
$$



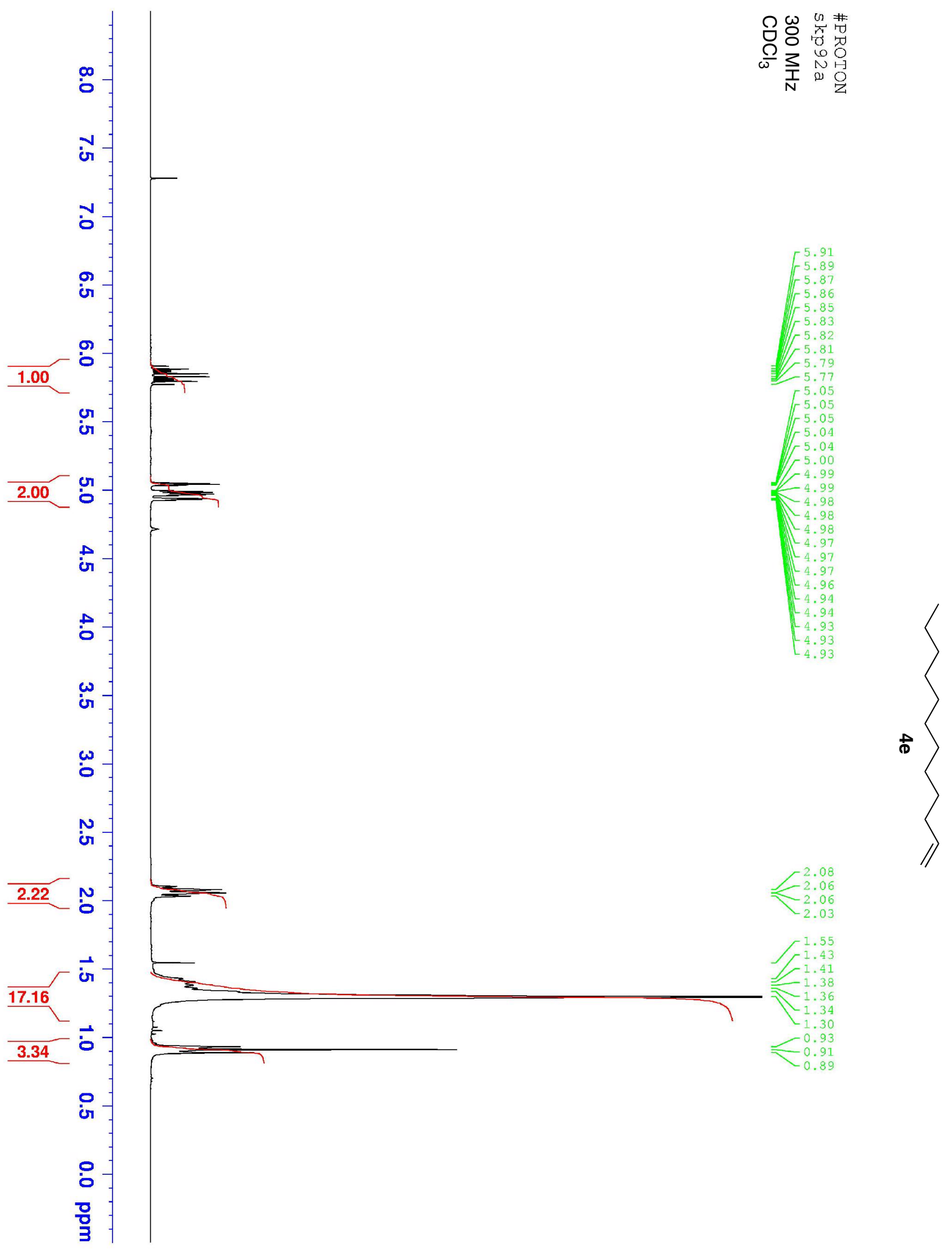


$$
1
$$




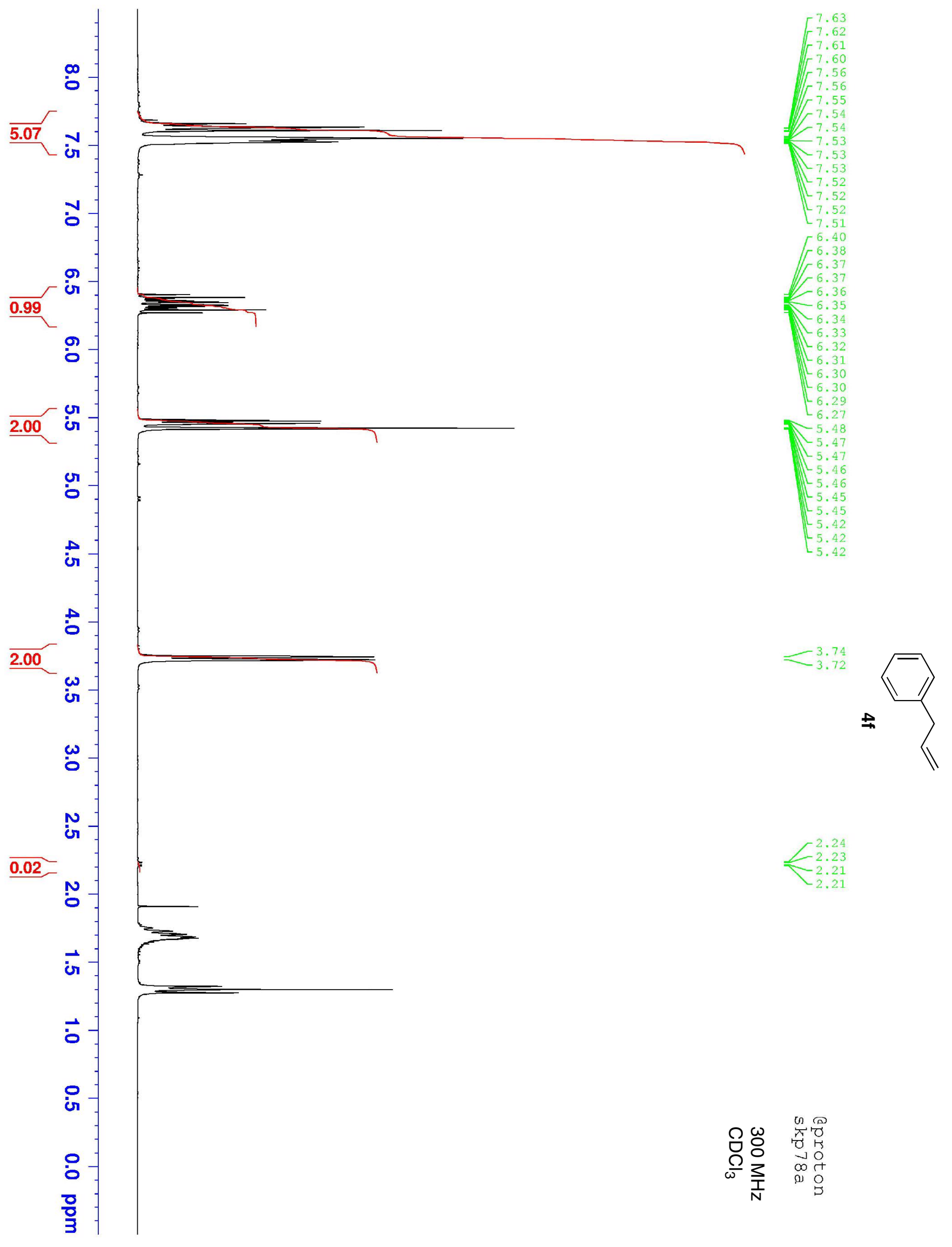




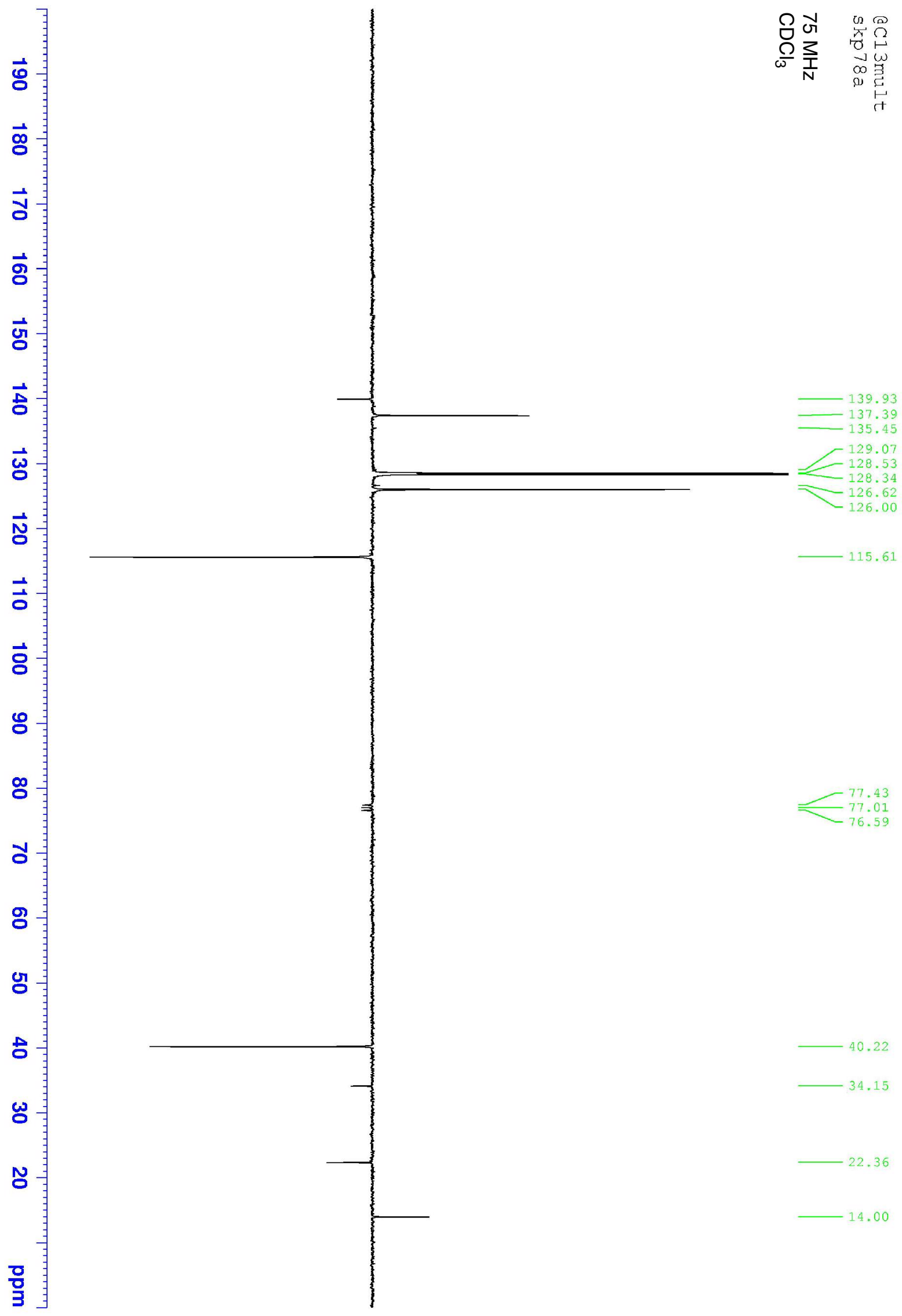

